\title{
The analgesic efficacy of dexamethasone as a perineural adjunct for peripheral nerve block versus systematic administration: a systematic review and meta-analysis
}

\author{
Eric Albrecht, ${ }^{1}$ Kyle Robert Kirkham, ${ }^{2}$
}

1. Department of Anaesthesia, Lausanne University Hospital, Lausanne, Switzerland.

2. Department of Anaesthesia, Toronto Western Hospital, University of Toronto, Toronto, Canada.

\begin{abstract}
Background and Goal of Study
Perineural dexamethasone has gained popularity in regional anaesthesia to prolong analgesia duration, but its advantage over systemic administration is disputed. The objective of this meta-analysis was to compare the analgesic efficacy of both routes of administration during peripheral nerve blockade.
\end{abstract}

\section{Materials and Methods}

The methodology followed the PRISMA statement guidelines. The primary outcome was duration of analgesia analysed according to the type of local anaesthetic administered (bupivacaine or ropivacaine). Secondary outcomes included cumulative opioid consumption in intravenous morphine equivalents, pain scores and complications rates (neurologic complication, infection, hyperglycaemia).

\begin{abstract}
Results and Discussion
Eleven controlled trials, including 914 patients, were identified. Duration of analgesia was significantly increased with perineural dexamethasone versus systemic dexamethasone by a mean difference of $3 \mathrm{~h}(95 \% \mathrm{CI}: 1.4,4.5 \mathrm{~h}$; $\mathrm{p}=0.0001$ ). Subgroup analysis reveals that the duration of analgesia was increased by $21 \%$ with bupivacaine (mean difference: 4.0 hours; $95 \%$ CI: $2.8,5.2$ hours; $\mathrm{p}<0.00001$ ) and $12 \%$ with ropivacaine (mean difference: 2.0 hours; $95 \% \mathrm{CI}:-0.5$, 4.5 hours; $\mathrm{p}=0.11$; Table 1 ). The quality of evidence for our primary outcome was moderate according to the GRADE system. There were no significant differences in other secondary outcomes. No neurological complications or infections were reported. Glucose levels were not increased when dexamethasone was injected systemically, but this outcome was reported by only two trials.
\end{abstract}

\section{Conclusion}

There is therefore moderate evidence that perineural dexamethasone combined with bupivacaine but not ropivacaine slightly prolongs analgesia duration, without impact on other pain related outcomes when compared to systemic dexamethasone. Injection of perineural dexamethasone should be cautiously balanced in light of the off-label indication for this route of administration.

Figure 1. Duration of analgesia according to type of local anaesthetics (bupivacaine vs ropivacaine).

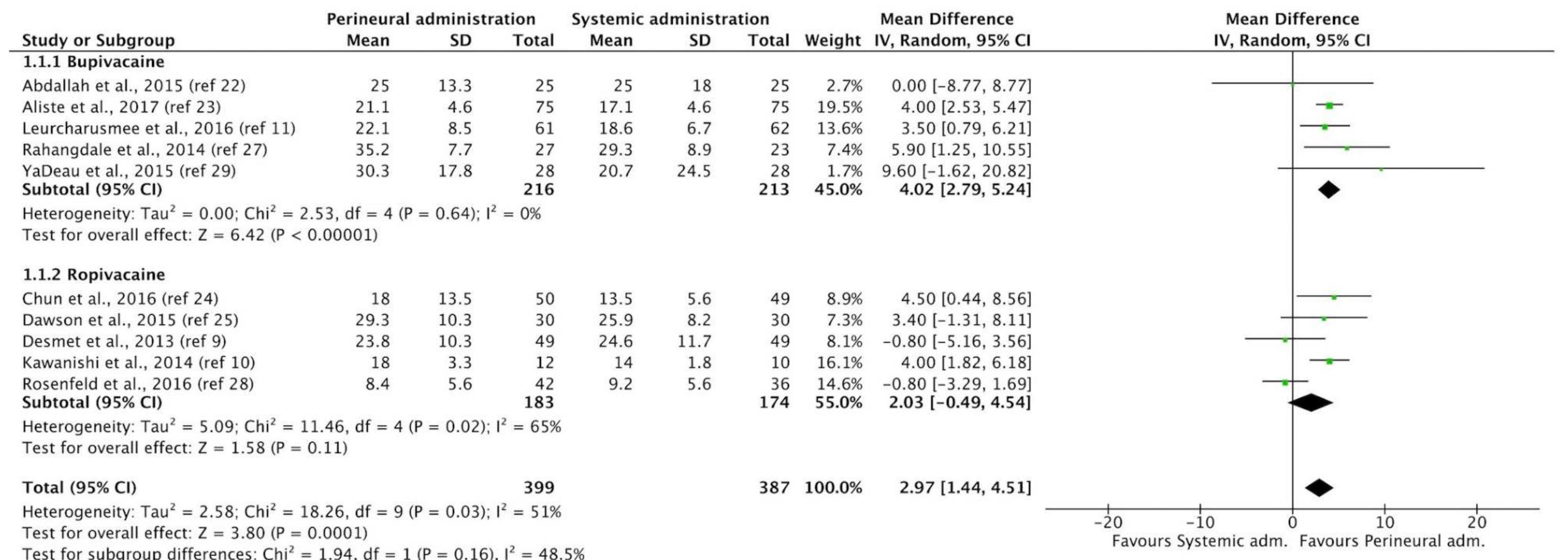

\section{References}

1. Axelsson K, Gupta A. Local anaesthetic adjuvants: neuraxial versus peripheral nerve block. Curr Opin Anaesthesiol 2009; 22: 649-54

2. Johansson A, Hao J, Sjölund B. Local corticosteroid application blocks transmission in normal nociceptive C-fibres. Acta Anaesthesiol Scand $1990 ; 34: 335-8$

3. Attardi B, Takimoto K, Gealy R, Severns C, Levitan ES. Glucocorticoid induced up-regulation of a pituitary K+ channel mRNA in vitro and in vivo. Receptors Channels 1993; 1: 287-93 